\title{
Đánh giá sự ảnh hưởng của hệ số ma sát đáy tới mực nước dâng do bão bằng mô hình ADCIRC khu vực Vịnh Bắc Bộ
}

\author{
Trần Thị Thảo, Hà Thanh Hương* \\ Khoa Khí tương Thủy văn và Hải dương học, Trường Đại học Khoa học Tụ nhiên, ĐHQGHN, \\ 334 Nguyến Trãi, Hà Nội, Việt Nam \\ Nhận ngày 08 tháng 8 năm 2016 \\ Chỉnh sửa ngày 26 tháng 8 năm 2016; Chấp nhận đăng ngày 16 tháng 12 năm 2016
}

\begin{abstract}
Tóm tắt: Các tính toán nước dâng do bão cho thấy ảnh hưởng của ma sát đáy là vô cùng quan trọng trong việc hiệu chỉnh thủy triều và là một trong những yếu tố quyết định sự chính xác của mô phỏng nước dâng do bão của mô hình. Bài báo này sử dụng modun ADCIRC thuộc hệ thống mô hình SMS để mô phỏng nước dâng do bão, trong đó khảo sát đến ảnh hưởng của hệ số ma sát đáy đến kết quả tính toán nước dâng thông qua việc biểu diễn hệ số ma sát đáy ứng với các trường hợp cho là hằng số tại các nút và trường hợp hệ số ma sát thay đổi tại các nút.Từ đó đưa ra việc xác định hệ số ma sát tối ưu áp dụng cho khu vực Vịnh Bắc Bộ để kết quả tính toán nước dâng do bão hiệu quả và nhanh nhất.
\end{abstract}

Tù khóa: Nước dâng do bão, ma sát đáy, ADCIRC.

\section{1. Đặt vấn đề}

Hệ số ma sát sử dụng trong mô hình $\mathrm{ADCIRC}$ thường là hằng số không đổi trên toàn miền tính. Việc sử dụng hệ số ma sát sẽ đạt được kết quả mực nước có độ chính xác cao khi tính toán cho một khu vực nhỏ có cùng chế độ thủy triều. Để tính toán cho kết quả tốt nhất người thường tìm các hệ số ma sát tương ứng tại các nút lưới. Tuy nhiên việc xây dựng một file hệ số ma sát tại các nút của miền tính lớn là rất khó khăn và tốn nhiều thời gian. Trong bài báo này tác giả đã tiến hành xác định độ nhạy của hệ số ma sát với các trường hợp tính toán khác nhau và so sánh kết quả tính toán mực

\footnotetext{
*Tác giả liên hệ. ĐT.: 84-912726027

Email: huonghat@vnu.edu.vn
}

nước với thực đo từ đó đưa ra hệ số ma sát phù hợp cho khu vực tính toán.

\section{Mô hình ADCIRC}

Mô hình ADCIRC được xây dựng và phát triển bởi các trường đại học bang Carolina bao gồm đại học Notre Dame, đại học Oklahoma và đại học Texas nước Mỹ. Mô hình ADCIRC (Advanced Circulation Model for Oceanic) là một hệ thống những mô hình giải các phương trình thủy động lực mô phỏng hoàn lưu tầng mặt và bài toán thủy động lực hai hoặc ba chiều [1]. Những chương trình này dùng phương pháp phần tử hữu hạn cho phép sử dụng các lưới phi cấu trúc có tính linh hoạt cao, đặc biệt có thể ứng dụng rất tốt cho các khu vực cửa sông ven biển có địa hình và đường bờ phức tạp. Mô 
hình được xây dựng dựa trên các phương trình cơ bản, bao gồm:

Phương trình liên tục:

$$
\frac{\partial \zeta}{\partial t}+\frac{\partial U H}{\partial x}+\frac{\partial V H}{\partial y}=0
$$

Các phương trình bảo toàn động lượng:

$$
\frac{\partial U}{\partial}+U \frac{\partial U}{\partial x}+V \frac{\partial U}{\partial y}-f V=\frac{\partial}{\partial x}\left[\frac{P}{P_{0}}+g \zeta-g(\eta+\gamma)\right]+\frac{\tau_{x x}}{P_{0} H}+\frac{\tau_{c x}}{P_{0} H}+D_{x}-B_{x}
$$$$
\frac{\partial V}{\partial t}+U \frac{\partial V}{\partial x}+V \frac{\partial V}{\partial y}+f U=\frac{\partial}{\partial y}\left[\frac{P}{P_{0}}+g \zeta-g(\eta+\gamma)\right]+\frac{\tau_{s y}}{P_{0} H}+\frac{\tau_{b y}}{P_{0} H}+D_{y}-B_{y}
$$

trong đó:

$\zeta$ là dao động mực nước $U, V$ là các vận tốc được lấy tích phân theo độ sâu theo hướng $\mathrm{x}$ và $\mathrm{y}$

$\mathrm{P}$ là áp suất, $\mathrm{H}$ là độ sâu mực nước

$\mathrm{Dx}$, Dy là các thành phần khuếch tán theo các phương

$\mathrm{Bx}$, By là các thành phần gradient áp suất theo các phương, $(\eta+\gamma)$ : thế thuỷ triều Newton, thuỷ triều trái đất và các lực mang bản chất lực thuỷ triều

$\tau_{b x},{ }_{b y}$ là ứng suất đáy theo các hướng $\mathrm{x}$ và y và $\mathrm{y}$

$\tau_{s x}, \tau_{s y}$ là ứng suất mặt theo các hướng $\mathrm{x}$

\section{$\rho_{0}$ là mật độ tham chiếu của nước}

\subsection{Hệ số ma sát trong $A D C I R C$}

Úng suất đáy trong phiên bản 2DDI của ADCIRC được biểu diễn như sau:

$$
\tau_{b x}=U \tau_{* \text { và }} \tau_{b y}=V \tau_{*}
$$

Tùy thuộc vào cách sử dụng, kết quả có thể là hàm tuyến tính, toàn phương hay tổ hợp của vận tốc trung bình theo độ sâu. Đối với hầu hết những ứng dụng cho vùng ven bờ, hàm ma sát toàn phương nên được sử dụng với hệ số ma sát $C_{f} \sim 0.0025$. Trong vùng nước rất nông, ma sát tổ hợp có hiệu quả hơn, với $C_{f m i n} \sim 0.0025$, đặc biệt khi các tham số khô và ướt được tính đến, khi đó biểu thức này giảm nhanh khi độ sâu nước trở nên nhỏ. Ma sát tuyến tính chủ yếu sử dụng cho thử nghiệm mô hình hoặc khi mô hình tuyến tính được chạy theo yêu cầu. Trong trường hợp này, độ lớn của $\tau_{*}$ phải phù hợp (ít nhất là về độ lớn) với giá trị được sử dụng tính toán biểu thức ma sát toàn phương và không phải với giá trị của $C_{f}$ thông thường được sử dụng trong biểu thức toàn phương [1] .

Trong trường hợp sử dụng hàm tuyến tính: $\tau_{*}=C_{f}$

trong đó $C_{f}=$ không đổi theo thời gian (có thể thay đổi theo không gian), đơn vị là s ${ }^{-1}$

Trong trường hợp sử dụng hàm toàn phương: $\tau_{*}=\frac{C_{f}\left(U^{2}+V^{2}\right)^{1 / 2}}{H}$

trong đó $C_{f}=$ không đổi theo thời gian (có thể thay đổi theo không gian), không thứ nguyên.

Hàm tổ hợp: $\quad H$

trong đó $C_{f}=C_{f \min }\left(1+\left(\frac{H_{\text {break }}}{H}\right)^{\theta}\right)^{\gamma / \theta}$ và $C_{f \min }, H_{\text {break (?)' }}, \gamma_{\text {(?) }}$ : không đổi theo thời gian.

Trong quan hệ ma sát tổ hợp, tiệm cận trong vùng nước sâu $\left(\mathrm{H}>\mathrm{H}_{\text {break }}\right)$ và tiệm cận với $C_{f \text { min }}\left(\frac{H_{\text {break }}}{H}\right)^{\gamma}$ trong vùng nước nông $(\mathrm{H}<$ $\left.\mathrm{H}_{\text {break }}\right)$. Số mũ $\theta$ xác định mức độ $C_{f}$ tiến đến giới hạn tiệm cận và $\gamma$ xác định mức tăng hệ số ma sát khi độ sâu giảm. Nếu $C_{f \text { min }}=g n^{2} / H_{b r e a k}^{\gamma}$ và $=1 / 3$, trong đó $g$ là gia tốc trọng trường và $n$ là hệ số Meaning, ma sát tổ hợp sẽ có một phương trình Meaning xử lý ma sát cho trường hợp $\mathrm{H}<H_{\text {break }}$. Ví dụ về mối quan hệ giữa $C_{f \min }$ và $\mathrm{n}$ khi $\gamma=1 / 3$ được đưa ra dưới đây. 
Bảng 1. So sánh giữa $C_{f \min }$ và n thì $C_{f \min }=g n^{2} / H_{\text {break }}^{\gamma}$

\begin{tabular}{lllll}
\hline$C_{f \text { min }}$ & $\mathrm{n}$ & $\mathrm{n}$ & $\mathrm{n}$ & $\mathrm{N}$ \\
\hline & $H_{\text {break }}=1 \mathrm{~m}$ & $H_{\text {break }}=5 \mathrm{~m}$ & $H_{\text {break }}=10 \mathrm{~m}$ & $H_{\text {break }}=20 \mathrm{~m}$ \\
0.0015 & 0.012 & 0.016 & 0.018 & 0.02 \\
0.002 & 0.014 & 0.019 & 0.021 & 0.024 \\
0.0025 & 0.016 & 0.021 & 0.023 & 0.026 \\
0.003 & 0.017 & 0.023 & 0.025 & 0.029 \\
0.004 & 0.02 & 0.026 & 0.03 & 0.033 \\
0.005 & 0.023 & 0.03 & 0.033 & 0.037 \\
0.01 & 0.032 & 0.042 & 0.047 & 0.053 \\
\hline
\end{tabular}

2.2. Điều kiện ban đầu và và điều kiện biên áp dụng trong mô hìn

Điều kiện biên mực nước

Điều kiện biên mực nước có thể được xác định thông qua 2 cách:

- Thứ nhất: mô hình tự nội suy các hằng số điều hòa tại mỗi nút biên từ bộ hằng số điều hòa toàn cầu và tính toán chuỗi mực nước tại mỗi nút biên.

- Thứ hai: từ các chuỗi mực nước theo thời gian tại mỗi nút biên.

Điều kiện thông lượng

ADCIRC cho pháp xác định các điều kiện biên bao gồm thông lượng pháp tuyến trên đơn vị rộng (nghĩa là thông lượng vuông góc bằng không tại các đoạn biên đất và khác không tại các đoạn biên sông). Quy tắc được dùng trong ADCIRC là thông lượng pháp tuyến đưa vào là thông lượng đi vào miền tính là dương và thông lượng đi ra là âm. Nếu thông lượng pháp tuyến được áp dụng như một điều kiện biên tự nhiên, sẽ không làm thay đổi các phương trình động lượng. Còn nếu điều kiện này được áp dụng như một điều kiện biên cần thiết, vận tốc pháp tuyến trung bình độ sâu $U_{N}$, là lực để cân bằng với thông lượng pháp tuyến chia cho độ sâu và nhân với -1 (?) (quy ước $U_{N}$ dương theo hướng vuông góc ra xa miền tính).

Điều kiện gradient vận tốc pháp tuyến bằng không tại biên.

$$
\begin{aligned}
& \frac{\partial U}{\partial N}=0 \quad \text { tại các nút trên biên. } \\
& \frac{\partial V}{\partial N}=0
\end{aligned}
$$
2DDI

Điều kiện biên vận tốc phát xạ - ADCIRC

Điều kiện biên phát xạ với mực nước được thực hiện bằng việc xác định mối liên hệ giữa vận tốc pháp tuyến và trường mực nước dọc biên.

Điều kiện gradient mực nước pháp tuyến bằng không tại biên

$$
\frac{\partial \zeta}{\partial n}=0
$$
2DDI

Điều kiện biên phát xạ trên mặt - ADCIRC

Điều kiện biên phát xạ với mực nước có thể được thực hiện bằng việc xác định mối liên hệ giữa thông lượng pháp tuyến và trường mực nước dọc biên.

\subsection{Các điều kiện triển khai cho mô hình}

Để triển khai mô hình ADCIRC tính toán mực nước dâng do bão với lưới tính toàn Biển Đông tại biên cứng, sử dụng điều kiện dính không thấm, các thành phần vận tốc lấy bằng 0 , Sử dụng điều kiện biên dao động mực nước tại các biên lỏng. Dao động mực nước thủy triều tại các biên lỏng được được tạo bằng mô đun TIDE từ hằng số điều hòa của 4 sóng $\mathrm{K} 1, \mathrm{O} 1$, M2, S2. Hệ số ma sát sử dụng trong mô hình được lấy theo các trường hợp tính toán là: 
- Hằng số không đổi cho toàn miền tính được lấy cho các trường hợp lần lượt là 0.003 , $0.004,0.0045$.

- Hệ số thay đổi theo các nút trên toàn miền tính được xây dựng và ghi định dạng theo file fort.21. Trong đó file fort.21 được xây dựng theo công thức tính hệ số ma sát đáy sử dụng trong mô hình Pom [2]. Hệ số ma sát được giới hạn cho Biển Đông trong khoảng [0.0025; 0.75]. Dựa vào độ sâu được nội suy từ file lưới tính thực hiện tính ma sát tại từng nút lưới theo công thức dưới đây:

$$
c b c=\frac{k^{2}}{\log \left[\frac{z s h+(1 . e 0+z z) D}{z 0 b}\right]^{2}}
$$

trong đó:

$\mathrm{k}$ là hằng số Von Karman's bằng 0.4

z0b là độ nhám đáy $(\mathrm{m})$ bằng 0.7

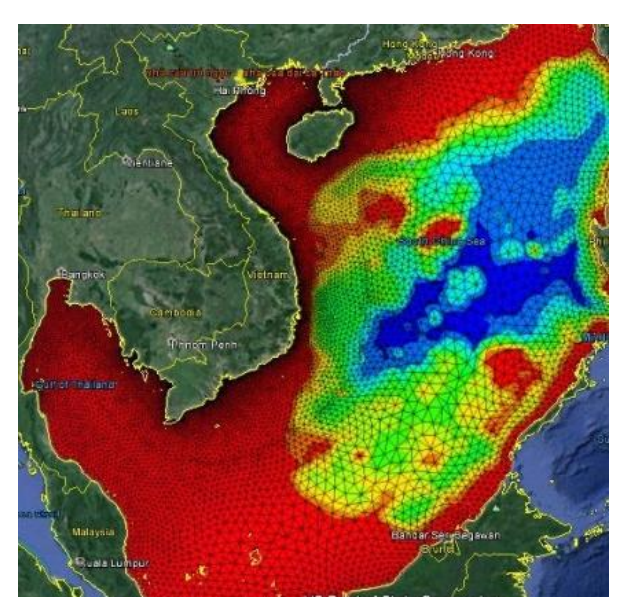

Hình 1. Lưới tính toàn biển Đông. zsh là độ trượt lớp biên đáy theo hàm Logarit $(\mathrm{m})$ bằng 0.01

$\mathrm{D}$ là độ sâu tại nút lưới

\section{Kết quả tính toán kiểm tra độ nhạy của hệ số ma sát đến nước dâng do bão}

Lưới tính sử dụng để khảo sát là lưới phi cấu trúc cho toàn vùng Biển Đông giới hạn từ $99^{\circ} \mathrm{E}-121^{\circ} \mathrm{E}$ và $1^{\circ} \mathrm{N}-25^{\circ} \mathrm{N}$ và khoảng cách lưới thưa ở các biên phía Đông, phía Nam và mịn dần về phía bờ biển Việt Nam. Khoảng cách lưới tính nhỏ nhất ở ven bờ phía vịnh Bắc Bộ và Bắc Trung Bộ là $5 \mathrm{~km}$. Các kết quả tính toán được trích xuất và so sánh với mực nước thực đo tại các trạm Hòn Dáu và Hòn Ngư trong bão Wukong (2000).

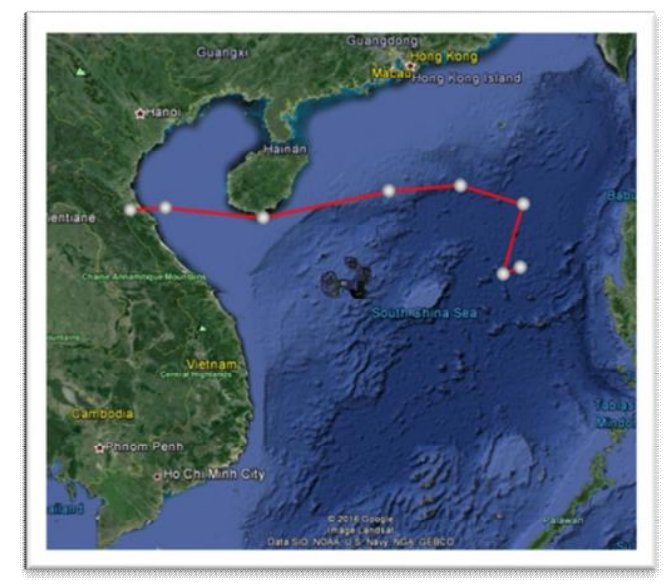

Hình 2. Quỹ đạo bão Wukong (2000).

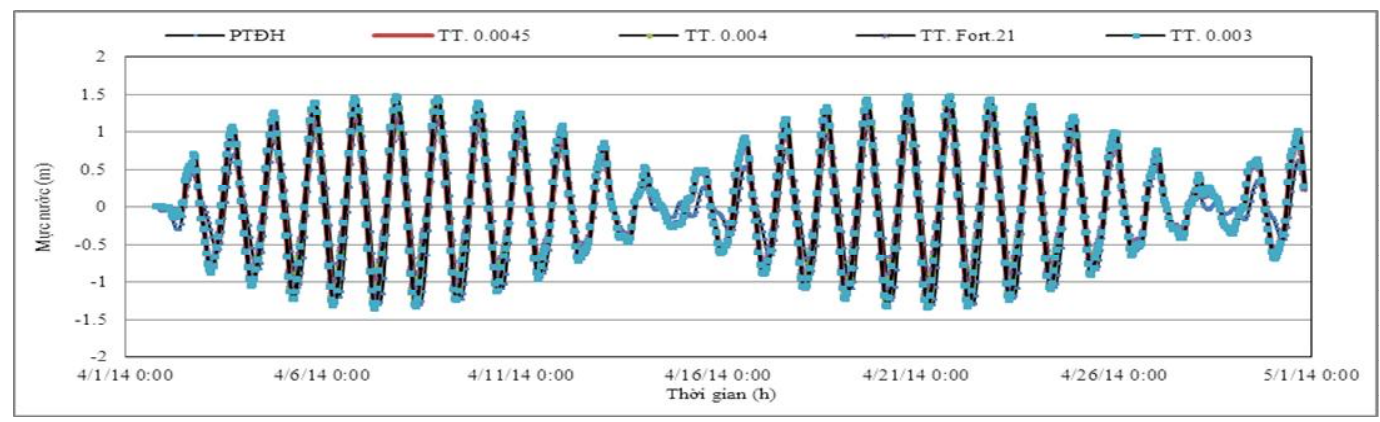

Hình 3. Kết quả nghiệm triều tại Hòn Dáu (phân tích điều hòa và tính toán). 


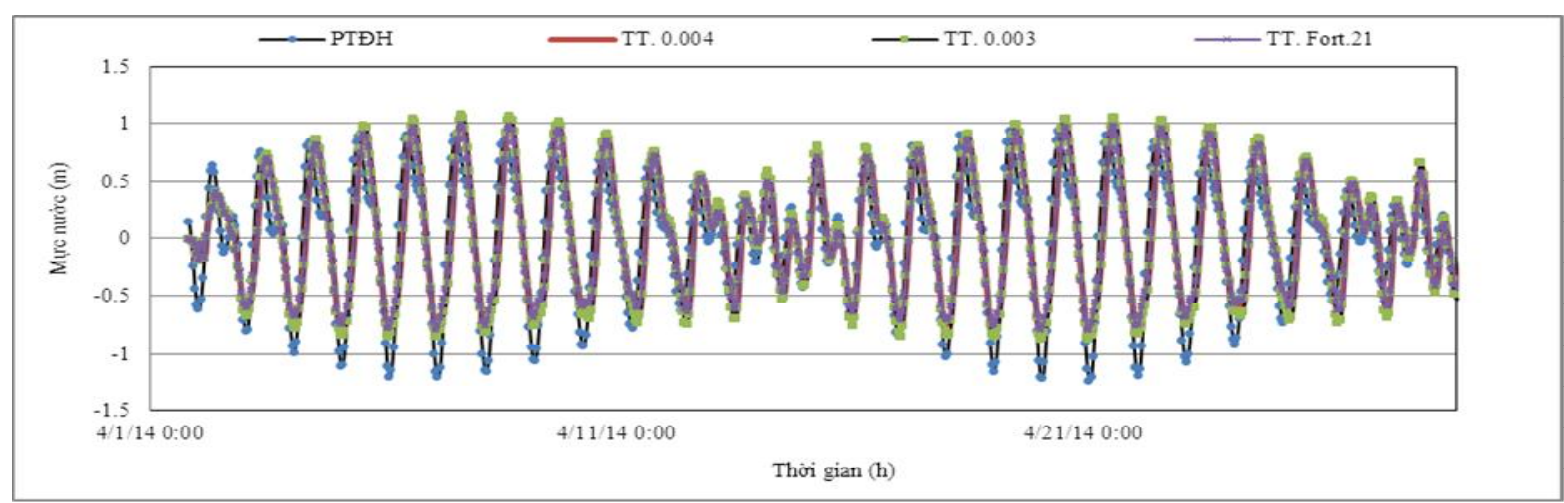

Hình 4. Kết quả nghiệm triều tại Hòn Ngư.

Bảng 2. Sai số tuyệt đối của các trường hợp tính toán thủy triều

\begin{tabular}{lllll}
\hline \multirow{2}{*}{ Trạm } & \multicolumn{4}{l}{ Sai số tuyệt đối so sánh với phân tích điều hòa thủy triều [?] } \\
\cline { 2 - 5 } & TT 0.0045 & TT 0.004 & TT 0.003 & TT Fort.21 \\
\hline Hòn Dáu & 0.206 & 0.252 & 0.316 & 0.246 \\
Hòn Ngư & 0.569 & 0.229 & 0.138 & 0.225 \\
\hline
\end{tabular}

\subsection{Hiệu chỉnh và kiểm chứng thủy triều}

Để khảo sát độ chính xác của mực nước thủy triều, thực hiện hiệu chỉnh thủy triều tại 2 trạm Hòn Dáu, Hòn Ngư, sau đó hiệu chỉnh với số liệu trích từ bảng thủy triều của Trung tâm Hải văn (Tổng cục Biển và Hải đảo). Hiệu chỉnh dao động mực nước thủy triều tính toán bằng cách thay đổi giá trị hệ số ma sát đáy.

Chuỗi mực nước thủy triều được tính từ $14 \mathrm{~h}$ ngày $01 / 04 / 2014$ đến 23 h ngày 29/04/2014. Chuỗi dao động mực nước tại các biên lỏng làm điều kiện biên của mô hình đã được hiệu chỉnh từ giờ UTC về múi giờ Hà Nội. Với tất cả các trường hợp của hệ số ma sát đáyđược đưa ra ở trên kết quả hiệu chỉnh triều đều cho kết quả khá tốt về pha và biên độ dao động thủy triều (Hình 3, 4).

Từ bảng tính toán sai số tuyệt đối của thủy triều tại trạm Hòn Dáu và Hòn Ngư trong các trường hợp hệ số ma sát khác nhau so sánh với phân tích điều hòa thủy triều cho thấy sai số tuyệt đối trong trường hợp hệ số ma sát là hằng số và bằng 0.004 cho giá trị nhỏ nhất cho cả 2 trạm và có giá trị xấp xỉ sai số tuyệt đối trong trường hợp hệ số ma sát thay đổi giữa các nút (fort.21). Điều này chứng tỏ các thông số của mô hình đã được hiệu chỉnh phù hợp với khu vực tính toán và có thể tiến hành tính toán nước dâng do bão.

\subsection{Kết quả tính toán kiểm tra độ nhạy của hệ số ma sát đến nước dâng do bão}

Để tiến hành kiểm tra độ nhạy của hệ số ma sát đến mực nước dâng do bão tác giả thực hiện tính toán nước dâng do bão Wukong (2000) theo 3 trường hợp với hệ số ma sát đáy không đổi và trường hợp ma sát đáy thay đổi tại các nút của miền tính với số liệu thực đo. Chuỗi số liệu khí tượng kéo dài 7 ngày từ $13 \mathrm{~h}$ ngày 04/09/2000 đến 18 h ngày 10/09/2000 [3].

Bão Wukong bắt đầu ảnh hưởng đến dải ven bờ Việt Nam vào $0 \mathrm{~h}$ ngày $8 / 9 / 2000$.Bão bắt đầu ảnh hưởng đến khu vực ven bờ với sức gió $10 \mathrm{~m} / \mathrm{s}$. Lúc này, nước dâng tại khu vực Bắc vịnh Bắc Bộ và dải ven bờ Bắc Trung Bộ cao khoảng $1.5 \mathrm{~m}$ do kết hợp với pha thủy triều lên. [Nên có hình vẽ minh họa]. 


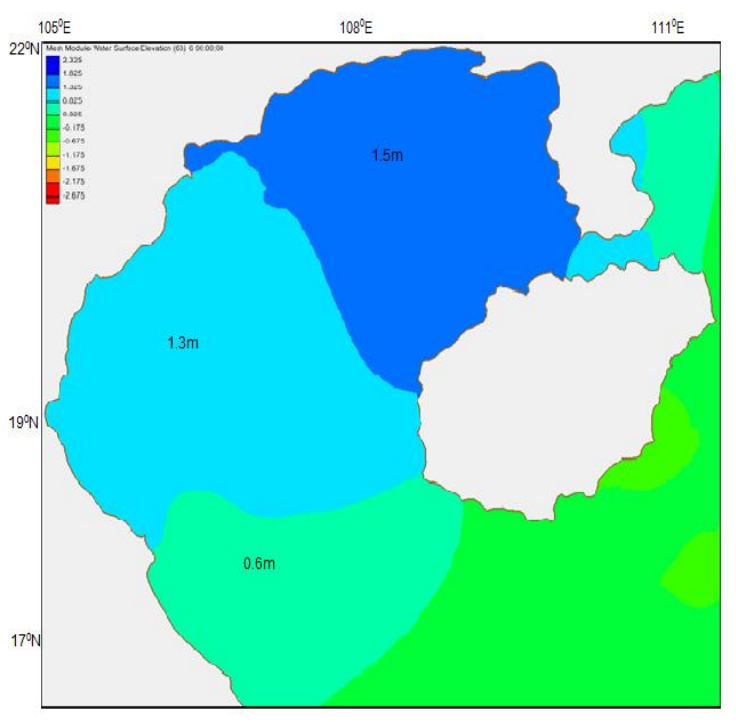

Hình 5. Thời điểm nước dâng cao nhất tại dải ven bờ lúc 22h ngày 09/09/2000.

Thời điểm bão bắt đầu đổ bộ vào tỉnh Hà Tĩnh lúc 22h ngày 9/9/2000. Mực nước dâng tại toàn bộ dải ven bờ từ Bắc Hà Tĩnh đến Quảng Ninh cao khoảng $1.5 \mathrm{~m}$ (Hình 5). Khu vực ven biển từ Đà Nẵng đến phía Nam Hà Tĩnh mực nước dâng cao khoảng $1.3 \mathrm{~m}$. Thời điểm bão bắt đầu đổ bộ rơi vào thời điểm pha triều xuống nên mực nước dâng toàn vùng thấp hơn thời điểm bão bắt đầu ảnh hưởng đến dải ven bờ

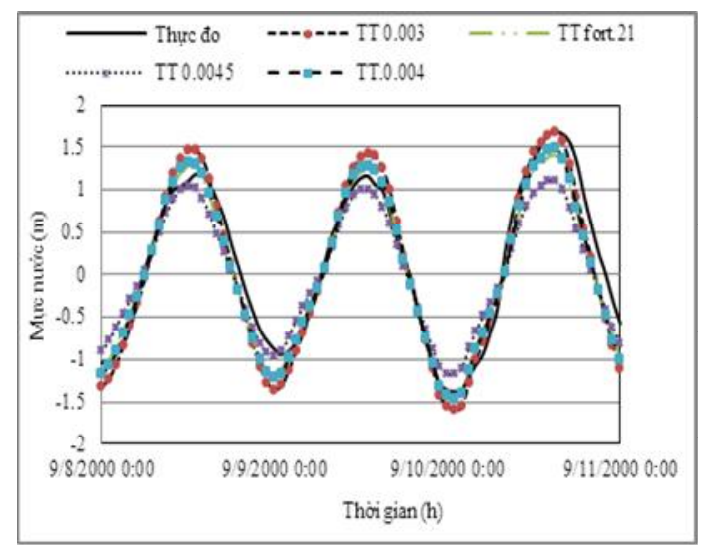

Hình 6. Biến trình nước dâng do bão các trường hợp tính toán và thực đo tại trạm Hòn Dáu.
Có thể thấy rằng thay đổi hệ số ma sát cho từng trường hợp tính toán cho kết quả tính toán mực nước chênh lệch nhau khá lớn. Đối với trường hợp tính toán hệ số ma sát bằng 0.003 khi so sánh với mực nước thủy triều cho chênh lệch lớn $(0.31 \mathrm{~m})$ nhưng kết quả tính toán mực nước dâng bão có sai số tuyệt đối khá nhỏ $(0.18 \mathrm{~m})$ và chỉ lớn hơn các trường hợp tính toán khác 2-3 cm tại Hòn Dáu.

Tại trạm Hòn Ngư do bão Wukong đổ bộ vào thời kỳ triều cường nên ảnh hưởng của thủy triều đến mực nước dâng do bão lớn. Việc thay đổi hệ số ma sát trong các trường hợp tính toán cho kết quả chênh lệch nhau khá lớn cả về hiểu chỉnh thủy triều và nước dâng do bão do chế độ thủy triều của khu vực này là triều hỗn hợp độ lớn $0.5-1 \mathrm{~m}$. Tuy nhiên các kết quả tính toán nước dâng lại bắt rất tốt về pha và thời gian nước dâng do bão. Sai số tuyệt đối của nước dâng dao động từ $0.35 \mathrm{~m}$ đến $0.28 \mathrm{~m}$ cao hơn rất nhiều so với trạm Hòn Dáu. Từ bảng 2 có thể thấy rằng việc lựa chọn hệ số ma sát đặc trưng cho cả vùng Vịnh Bắc Bộ là 0.004 (TT 0.004) cho sai số nhỏ và gần với trường hợp lựa chọn hệ số ma sát tính toán tại các nút lưới (TT Fort.21). Vì vậy trong trường hợp cần dự báo nhanh nước dâng do bão ta có thể áp dụng hệ số ma sát là hằng số cho toàn vịnh.

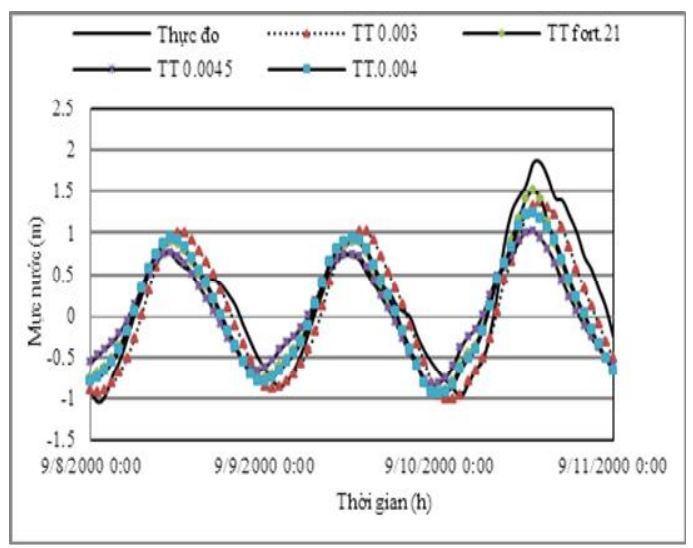

Hình 7. Biến trình nước dâng do bão các trường hợp tính toán và thực đo tại trạm Hòn Ngư. 
Bảng 3. Sai số tuyệt đối của các trường hợp tính toán nước dâng

\begin{tabular}{lllll}
\hline \multirow{2}{*}{ Trạm } & \multicolumn{4}{l}{ Sai số tuyệt đối so sánh với mực nước thực đo } \\
& TT 0.0045 & TT 0.004 & TT 0.003 & TT Fort.21 \\
\hline Hòn Dáu & 0.154 & 0.169 & 0.188 & 0.169 \\
Hòn Ngư & 0.349 & 0.287 & 0.226 & 0.285 \\
\hline
\end{tabular}

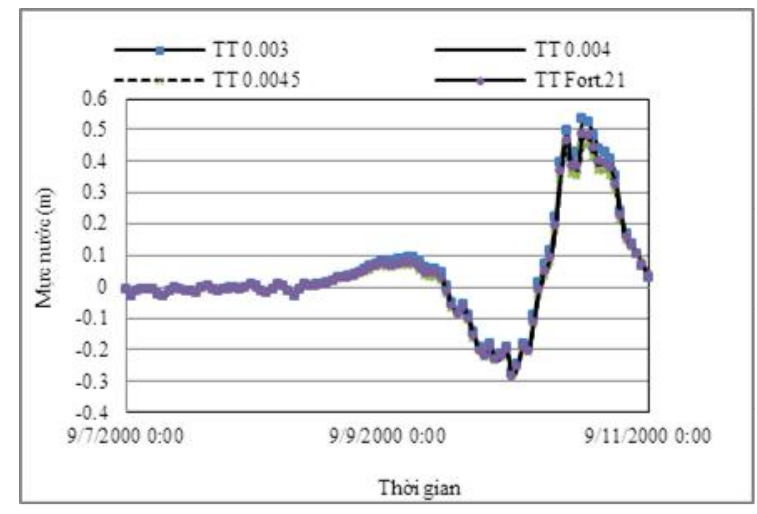

Hình 8. Biến trình nước dâng do gió bão trong các trường hợp tính toán tại trạm Hòn Ngư (không xét đến ảnh hưởng của thủy triều).

Bão Wukong đổ bộ vào thời kỳ triều cường nên ảnh hưởng của thủy triều đến mực nước dâng do bão lớn. Mực nước dâng tổng cộng lên đến hơn $1.5 \mathrm{~m}$ nhưng khi không tính đến ảnh hưởng của thủy triều, mực nước dâng do bão vào thời điểm cao nhất chỉ cao khoảng $0.5 \mathrm{~m}$. Tại thời điểm bão chưa ảnh hưởng tới khu vực ven bờ, tất cả các trường hợp tính toán khác nhau của hệ số ma sát đều cho một kết quả mực nước (các đường trùng khít nhau), chỉ khi bảo ảnh hưởng tới khu vực ven bờ (từ $15 \mathrm{~h}$ ngày 08/9/2000) mới có sự thay đổi về mực nước giữa các trường hợp tính toán (hình 8 ) chênh lệch mực nước dâng cao nhất giữa các trường hợp tính toán khoảng $10 \mathrm{~cm}$.

\section{Kết luận}

Từ các kết quả tính toán cho thấy sự thay đổi của hệ số ma sát ảnh hưởng lớn đến kết quả tính toán nước dâng do bão khu vực Vịnh Bắc Bộ. Tác giả đã kiểm nghiệm và lựa chọn được hệ số ma sát bằng 0.004 áp dụng cho toàn khu vực Vịnh Bắc Bộ thay vì phải xác định hệ số ma sát tại các nút lưới tính và cho ra kết quả có sai số tuyệt đối giữa hai trường hợp này là gần tương đương.

\section{Tài liệu tham khảo}

[1] A (parallel) advanced circulation model for oceanic, coastal and estuarine waters.

[2] Pom08.f - Center for Coastal Physical Oceanography.

[3] Typhoon 200016 (Wukong)-General Information (Pressure and Track Charts). 


\title{
Assessment the Effects of Bottom Friction Coefficient to Storm Surge Water Levels by Model ADCIRC in the Gulf of Tonkin Region
}

\author{
Tran Thi Thao, Ha Thanh Huong \\ Faculty of Hydro-Meteorology and Oceanography, College of Science, VNU University of Science, \\ 334 Nguyen Trai, Hanoi, Vietnam
}

\begin{abstract}
The storm surge calculations show that the effect of bottom friction is extremely important to calibrate the tide and is one of the determinants of the accuracy of the storm surge simulation bymodels. In this paper modules ADCIRC of SMS model system is used to simulate the storm surge for assessment the effect of bottom friction coefficient to storm surges calculation results through cases with constant bottom friction coefficient and that coefficient to be calculated at every nodes of the grid.The optimum friction coefficients has selected and applied to the Tonkin Gulf region, that makes the calculated results storm surges to be more effective
\end{abstract}

Keywords: Storm surge water, Bottom Friction, ADCIRC. 